\title{
Noise Quantification and Noise Reduction for Unipolar and Bipolar Electrograms
}

\author{
Laura A Unger ${ }^{1}$, Tobias G Oesterlein ${ }^{2}$, Axel Loewe $^{1}$, Olaf Dössel ${ }^{1}$ \\ ${ }^{1}$ Institute of Biomedical Engineering, Karlsruhe Institute of Technology (KIT), Karlsruhe, Germany \\ ${ }^{2}$ Boston Scientific, Ratingen, Germany
}

\begin{abstract}
Intracardiac electrograms (EGMs) form the basis for the diagnosis of arrhythmia mechanisms. Bipolar EGMs dominate clinical practice despite major disadvantages over unipolar EGMs since noise strongly distorts the latter.

In this study, we quantified and reduced the noise level of uni- and bipolar EGMs recorded with Rhythmia HDx and the Orion catheter. Distinct noise frequencies in the power spectral density (PSD) were detected with a sliding window of variable width and subsequently removed by notch filtering. The absolute peak to peak voltage remaining in the inactive segments after baseline removal quantified the noise level of the system. An international, multi-center selection of 33 patients served as a broad sample cohort.

The case-specific detection and removal of noise peaks reduced the noise level in unipolar EGMs by $30 \%$ to $0.076 \mathrm{mV}$ compared to standard clinical filtering. With a bipolar noise level of $0.01 \mathrm{mV}$, we saw that Rhythmia $H D x$ meets the low noise floor claimed in the system specifications. Certain noise frequencies presented permanently in all cases whereas others showed up only intermittently or in individual cases.

The suggested extension of filter settings lowers the noise level, enhances the detailed segmentation of low voltage areas, and encourages to exploit the advantages of unipolar over bipolar EGMs in clinical practice.
\end{abstract}

\section{Introduction}

The analysis of intracardiac EGMs is a cornerstone in deducing arrhythmia mechanisms during electrophysiological studies. Both visual review by experienced electrophysiologists and computation of EGM features are highly dependent on the signal quality. However, undesirable components such as high and low frequency noise, the ventricular far field, power line hum, and other parasitic frequencies overlay the EGM of interest. Various techniques have been proposed to eliminate disturbances among which the calculation of bipolar leads has evolved to be the clinical standard [1]. Far field noise occurring equally in both unipolar leads of a bipolar pair is automatically removed by subtraction. Combining two unipolar leads yet implicates loss of spatial accuracy and morphological features and introduces dependencies on the direction of excitation propagation [2]. Despite overcoming these disadvantages, unipolar EGMs are rarely used due to a lack of efficient noise removal methods. Opposed to bipolar EGMs, even the removal of far field noise is demanding since the components do not simply cancel out. The three-dimensional electroanatomical mapping system (EAMS) Rhythmia HDx claims to lower the bipolar noise level down to $0.01 \mathrm{mV}$ [3] with the IntellaMap Orion catheter (Boston Scientific, Malborough, USA) and thus to reveal low voltage EGM components which are of particular interest for a detailed diagnosis. Information on the noise level of an EAMS is crucial to understanding its strengths but also its limitations regarding the minimally resolvable voltage amplitude. In this study, we systematically quantified the noise level of both unipolar and bipolar EGMs recorded with the IntellaMap Orion catheter in a multi-center study. In a second step, we further developed a method for removing distinct noise peaks in frequency domain and assessed the resulting reduction of noise levels.

\section{Methods}

\subsection{Clinical study cohort}

Patients with stable atrial tachycardia and unequivocal driving mechanism were extracted from the international, multi-center TrueHD database (Boston Scientific, Malborough, USA) of ultra-high-density mapping gathered with the IntellaMap Orion catheter using the Rhythmia HDx EAMS. A total of 33 patients from 17 centers met the above-mentioned inclusion criteria. For each case, an area of reference tissue was annotated. Reference tissue was required to exhibit bipolar voltage amplitudes larger than the clinical standard of $0.5 \mathrm{mV}$ and needed to be passed by a planar wavefront with physiological conduction velocity. 


\subsection{Quantification of noise levels}

Distinguishing between noise and atrial activity is a nontrivial task for pathological EGMs of potentially low voltage amplitudes. However, for quantifying the noise level of an EGM segment, we needed to assure that the respective segment merely consisted of noise and was not exposed to possible other sources of EGM alteration. Using only EGMs that fulfilled the beat acceptance criteria of the EAMS and that were recorded within the annotated reference area assured reliable separability of active and inactive EGM segments since atrial activity complexes were expected to be narrow, sharp, and of high amplitude. Atrial activity was detected with the non-linear energy operator [4] in intervals of $2 \mathrm{~s}$ duration centered around each accepted beat. An EGM segment was considered active if the activity persisted for at least $10 \mathrm{~ms}$ and inactive in any other case. Post-processing merged active segments closer than $30 \mathrm{~ms}$. Additionally, QRS complexes were detected in Einthoven lead I of the surface ECG [5] and labelled as active for all EGM leads. For any remaining inactive segment, the baseline was removed using two different approaches: Fitting and subtracting a $5^{\text {th }}$ order polynomial or highpass filtering with a cutoff frequency of $30 \mathrm{~Hz}$. Both approaches were independently applied to the database and compared in the end. The resulting signals were considered to contain noise only. In order to prevent boundary effects from distorting the results, the onset and the offset of the remaining segments were cropped by $5 \mathrm{~ms}$ each. The maximum absolute peak to peak voltage of the inactive segment quantified the noise level conservatively.

\subsection{Removal of distinct noise frequencies}

The power spectral density (PSD) of recorded EGMs revealed distinct, narrow peaks exceeding their surrounding in amplitude, hence being considered noise. Atrial activity was also present in the recorded EGMs causing broader alterations in the PSD and thus not interfering with the noise peaks. Under the assumption that noise frequencies continuously overlaid the recordings during the entire intervention, we chose the longest consecutive EGM recording for determining noise frequencies in order to achieve the highest possible resolution in frequency domain. For a recording of length $\mathrm{T}$, the frequency resolution of the PSD is given by $\Delta f=\frac{1}{T}$. A sliding window of width $w$ was shifted along the PSD [6]. For each window position $f$ covering the interval $\left[f-\frac{w}{2} ; f+\frac{w}{2}\right)$, the maximum amplitude of the PSD within the sliding window was compared to the maximum amplitude within the consecutive intervals of width $20 \mathrm{w}$ to the left and the right of the central window. If the maximum spectral amplitude within the central sliding window exceeded the maximum amplitude within the consecutive windows by at least $6 \mathrm{~dB}$, the frequency exhibiting the prominent peak was considered noise. For frequencies larger than or equal to $45 \mathrm{~Hz}$, the peaks were removed with a notch filter of width $w$. The PSD of the filtered signal was recursively checked for peaks. The approach was then repeated for window widths $w$ increasing from 10 samples in frequency domain to a maximum width of $0.3 \mathrm{~Hz}$ in steps of 10 samples. Assuming that noise equally affects all electrodes of the Orion catheter, we determined peaks for one unipolar lead only and applied notch filtering to all leads. In order to distinguish between the power line hum and other noise frequencies, the peak between 45 and $65 \mathrm{~Hz}$ with the highest amplitude was considered the power line frequency $f_{P L}$ with corresponding harmonics if peaks were present at $n \cdot f_{P L}, n \in \mathbb{N}$.

\subsection{Filter settings}

For unipolar EGMs, clinical standard filtering with a highpass and lowpass cutoff frequency at $1 \mathrm{~Hz}$ and $300 \mathrm{~Hz}$ removed high and low frequency noise from the acquired EGM. The clinical standard filter setting also included the application of Gaussian notch filters to the power line frequency and its harmonics. An extended filter setting consisted of additional notch filters for the remaining set of detected noise frequencies for unipolar EGMs. Bipolar EGMs were only subject to clinical standard filtering with a high- and lowpass cutoff frequency of $30 \mathrm{~Hz}$ and $300 \mathrm{~Hz}$.

\section{Results}

\subsection{Noise frequencies in unipolar electro- grams}

Figure 1 visualizes the workflow for the quantification of the noise level on an exemplary basis. The absolute peak to peak voltage of the processed inactive signal was used as a measure for the noise level of the EGM segment.

Figure 2 shows the PSDs of the raw EGM recording, after clinical filtering, and after extended filtering. The power line frequency was detected at $60 \mathrm{~Hz}$ with corresponding harmonics. The clinical filter setting leaves the PSD with numerous distinct noise peaks that can be removed by extended filtering to increase signal quality.

Figure 3 depicts the clinical EGMs corresponding to the PSDs in Figure 2. The raw clinical recording is distorted by several noise frequencies. After clinical filtering, i.e. high- and lowpass filtering and removal of the power line frequency and corresponding harmonics, the noise level decreased. The extended filter setting yielded a clear EGM consisting of sharp atrial activities alternating with broader deflections caused by the ventricular far field.

In each of the 33 cases of the multi-center study cohort, we found distinct noise peaks at $80 \mathrm{~Hz}$ and the respective harmonics. Furthermore, noise was present at $120 \mathrm{~Hz}$, 

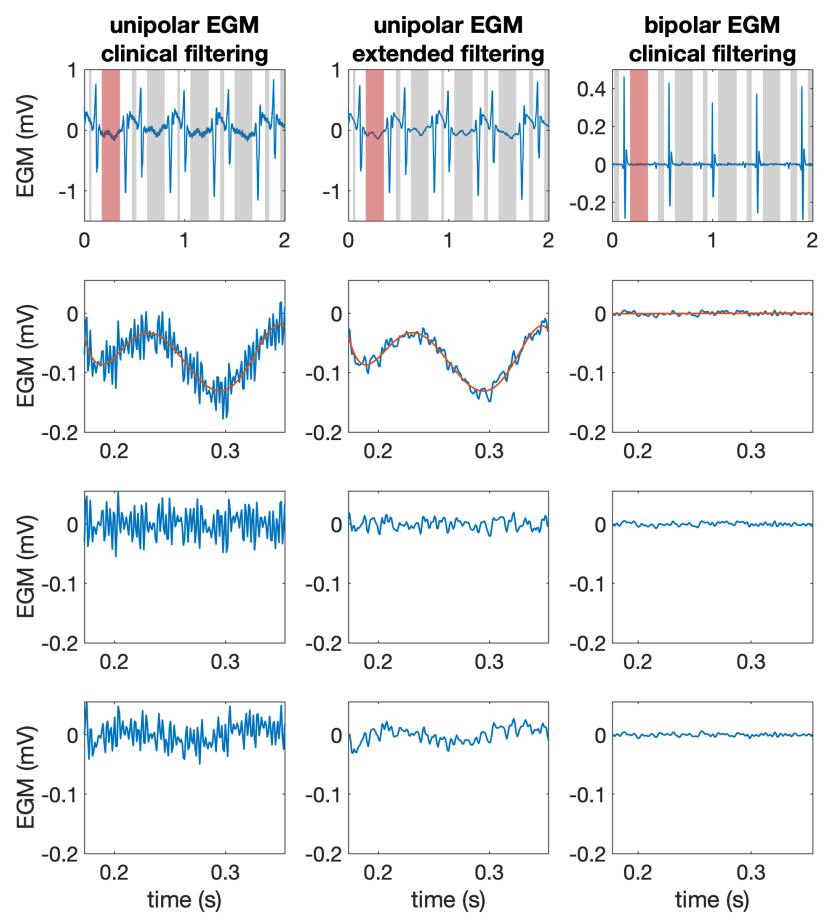

Figure 1. First row: EGM traces of $2 \mathrm{~s}$ length. Inactive segments are shaded in gray and red. Second row: Magnified representation of the inactive EGM segment shaded in red. The red curve depicts the polynomial baseline fit. Third row: Resulting inactive segment after polynomial baseline removal. Fourth row: Resulting inactive segment after baseline removal by highpass filtering. The three columns depict the clinically filtered unipolar EGM, the unipolar EGM after extended filtering, and the clinically filtered bipolar EGM, respectively.

$200 \mathrm{~Hz}, 360 \mathrm{~Hz}$, and $440 \mathrm{~Hz}$ for all analyzed cases. A set of additional frequencies was repeatedly detected in the majority of the cohort. Other noise frequencies occurred only in single cases.

\subsection{Noise level of Rhythmia HDx}

From the whole cohort, we extracted 40574 and 42321 inactive segments from the unipolar and bipolar EGMs recorded within the reference area, respectively. Figure 4 shows the median noise level as well as the $25 \%$ and $75 \%$ quantile for clinical and extended filter settings applied to unipolar EGMs, and the clinically filtered bipolar EGMs. For the highpass filtering approach for baseline removal, the median noise level for clinical and extended filter settings for unipolar EGMs as well as for clinical filter settings for bipolar EGMs measured $0.110 \mathrm{mV}, 0.076 \mathrm{mV}$, and $0.010 \mathrm{mV}$, respectively. For the polynomial baseline removal, the median noise level measured $0.105 \mathrm{mV}$,
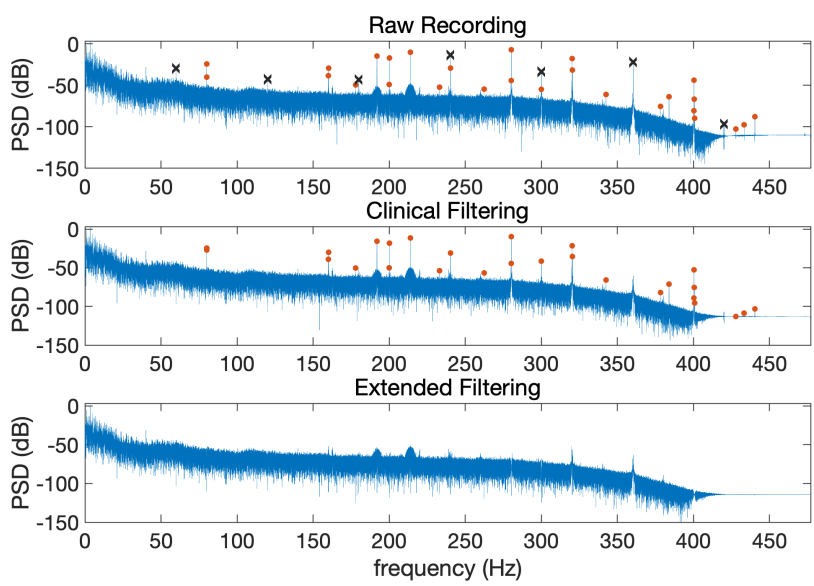

Figure 2. PSD of the raw unipolar EGM, after clinical filtering, and extended filtering. Black crosses mark the detected power line frequency and corresponding harmonics. Red dots label additional noise frequencies.
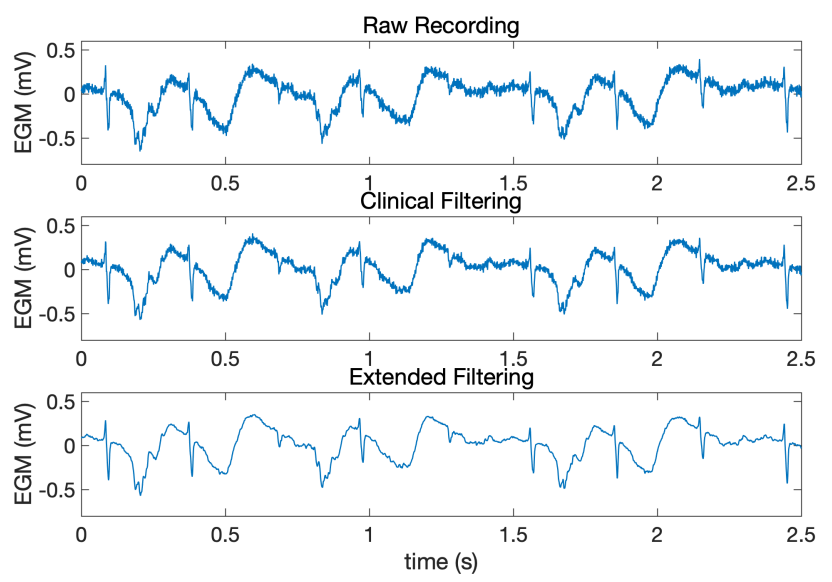

Figure 3. Raw unipolar EGM recording, EGM after clinical filtering and EGM after extended filtering. The EGM segment corresponds to the PSDs in Figure 2.

$0.062 \mathrm{mV}$, and $0.016 \mathrm{mV}$, respectively. Additional filtering reduced the noise level significantly ( $p<0.0001)$ as compared to the clinical filter settings.

\section{Discussion}

Unipolar EGMs are distorted by a multitude of noise frequencies. For a possible transfer of unipolar EGMs to clinical practice, effective filtering that exceeds the current clinical standard of only removing the power line frequency is inevitable. The proposed method of pinpointing distinct noise peaks in the PSD and subsequent removal from the EGMs proved to be effective.

Both postprocessing approaches for baseline removal yielded similar noise levels. The filtering approach was 


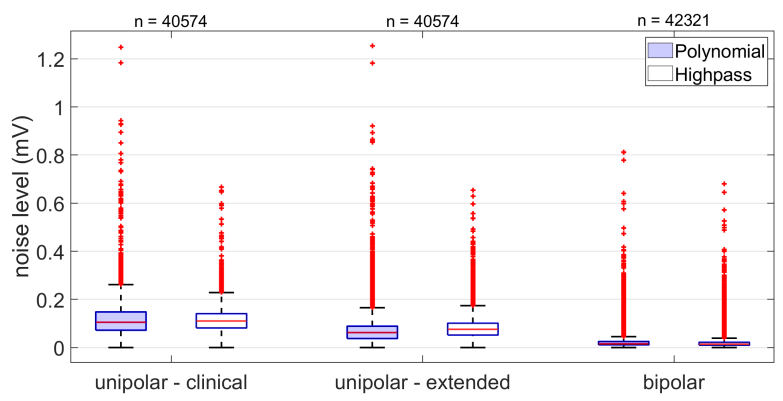

Figure 4. Noise levels of $n$ segments for unipolar EGMs after clinical and extended filtering as well as for bipolar EGMs. The baseline was removed by subtraction of a polynomial fit or $30 \mathrm{~Hz}$ highpass filtering. The red line marks the median noise level. The blue box surrounds the $25 \%$ and $75 \%$ quantile. Red crosses depict outliers beyond the $5 \%$ and $95 \%$ quantile as indicated by black bars.

more robust with little number of outliers but remnant lowfrequency oscillations, which caused the unipolar noise level to slightly exceed the results of the polynomial approach. The polynomial approach, on the other hand, decreased the remnant oscillations on the cost of an increasing number of outliers.

Deeper analysis revealed that, opposed to the assumptions made above, there are systematic inter-electrode differences in the PSD. In particular, the proximal electrodes 7 and 8 were susceptible to additional noise frequencies. However, this work did not further investigate interelectrode variations of the PSD but applied the same filter settings to all leads. Electrode specific filtering will need to be investigated in a follow-up study. We also saw time dependent occurrences of certain noise frequencies. In order to account for the temporal dynamics of noise, the spectral analysis needs to involve a temporally resolved component. Instead of using a set of fixed window widths $w$ for peak detection in the PSD, the advantages determining the actual peak width from the PSD need further investigation. Center specific noise frequencies resulting from the individual setup of the electrophysiology laboratory could be quantified and used as background knowledge for the individual EAMS setup. Noise levels determined in this study are a conservative estimate of the actual noise level of the system. The presented workflow is expected to slightly overestimate the noise level since suboptimal baseline removal in the inactive segment, e.g., elevates the result. Therefore, the resulting noise levels can be considered an upper limit of expected additive noise amplitudes.

To conclude, we quantified the noise level for unipolar and bipolar EGMs recorded with the Rhythmia HDx EAMS and the IntellaMap Orion catheter and thus give insight into the minimally resolvable voltage amplitudes during an electrophysiological study. The measured bipo- lar noise level of $0.010 \mathrm{mV}$ meets the Rhythmia HDx system specification [3]. A low noise floor allows to further discriminate regions of low voltage to specify diagnosis. Furthermore we showed that carefully chosen extended filtering significantly improves the signal quality and should be applied to unipolar EGMs in clinical practice. Despite the lower noise level in bipolar leads, this study aims at enhancing the use of unipolar leads and encouraging the community to leverage morphological and spatially resolved features that exceed the information content of bipolar EGMs.

\section{Acknowledgments}

We gratefully acknowledge the support by Annika Haas and Armin Luik during clinical case review. Boston Scientific kindly provided the TrueHD database. The research leading to the presented results has received funding from Boston Scientific and the German Research Foundation (DFG) under grant DO637/23-1.

\section{References}

[1] Tedrow UB, Stevenson WG. Recording and interpreting unipolar electrograms to guide catheter ablation. Heart Rhythm May 2011;8(5):791-796.

[2] Schuler S, Keller MW, Oesterlein T, Seemann G, Dössel O. Influence of catheter orientation, tissue thickness and conduction velocity on the intracardiac electrogram. Biomedical Engineering Biomedizinische Technik Sep 2013;58(s1).

[3] Rhythmia HDx Mapping System CLARITY for any level of complexity. https://www.bostonscientific.com/content/dam/bostonscienti fic/ep/Electrophysiology2017. Online, accessed 2018-07-10.

[4] Nguyen MP, Schilling C, Dössel O. A new approach for automated location of active segments in intracardiac electrograms. In IFMBE Proceedings World Congress on Medical Physics and Biomedical Engineering, volume 25(4). 2009; 763-766.

[5] Lenis G, Pilia N, Oesterlein T, Luik A, Schmitt C, Dössel O. $P$ wave detection and delineation in the ECG based on the phase free stationary wavelet transform and using intracardiac atrial electrograms as reference. Biomedizinische Technik Biomedical Engineering Jul 2016;61(1):37-56.

[6] Huck S, Oesterlein T, Luik A, Schmitt C, Wakili R, Dössel O. Preprocessing of unipolar signals acquired by a novel intracardiac mapping system. Current Directions in Biomedical Engineering Sep 2016;2(1):259-262.

Address for correspondence:

Laura Anna Unger, MSc

Institute of Biomedical Engineering,

Karlsruhe Institute of Technology (KIT)

Kaiserstraße 12, 76131 Karlsruhe, Germany

publications@ibt.kit.edu 